\title{
ESTUDIO DE CASO: ANÁLISIS BIOMECÁNICO Y FISIOLÓGICO DEL SKYRUNNER ${ }^{\circledR}$
}

\author{
José Moncada Jiménez* \\ Docente e investigador de la Escuela de Educación Física y Deportes, \\ de la Universidad de Costa Rica \\ San José, Costa Rica \\ Pietro Scaglioni Solano* \\ Docente e investigador de la Escuela de Ingeniería Mecánica, \\ de la Universidad de Costa Rica \\ San José, Costa Rica
}

Recibido 19-VIII-2008 • Aceptado 18-IX-2008 • Corregido 5-VI-2009

\begin{abstract}
Resumen: El propósito de este estudio de caso fue analizar un implemento deportivo de venta en el mercado costarricense, el Skyrunner ${ }^{\circledR}$, desde el punto de vista biomecánico y fisiológico. Para ello, se estudiaron las diferencias biomecánicas y fisiológicas cuando se usa y no se usa el Skyrunner ${ }^{\circledR}$. Un hombre (20 años, 1,67 m, $55 \mathrm{~kg}$ ) fue el caso de estudio. El análisis de las variables biomecánicas se realizó por medio de una plataforma de fuerza, con el que se obtuvo la fuerza vertical, el tiempo de vuelo, la altura del salto, el impulso vertical y la duración de la fase de impulso. Para la medición de las variables fisiológicas se utilizó un carro metabólico para evaluar el gasto energético mientras el sujeto se ejercitaba en una banda sin fin durante el calentamiento, el ejercicio y la fase de recuperación. En general, la utilización del Skyrunner ${ }^{\circledR}$ provocó un mayor gasto de energía, que se puede atribuir al requerimiento de mecanismos de equilibrio y tensión muscular que conllevan un gasto importante de energía. La altura de los saltos con Skyrunner ${ }^{\circledR}$ fueron un 50\% más altas, a pesar de que el impulso vertical fue cerca de un $15 \%$ menor. El tiempo de impulsión fue de un $27,5 \%$ del tiempo de un salto normal y se generaron fuerzas casi tres veces mayores. Esto denota la gran eficiencia mecánica del sistema, que es capaz de guardar y utilizar mayor cantidad de energía elástica acumulada en la estructura, en comparación con la que producen las piernas en forma aislada.
\end{abstract}

Palabras clave: Metodología, fisiología, biomecánica, gasto energético, salto.

\section{Introducción}

Uno de los problemas más frecuentes que posee la población estudiantil del área de la Educación Física y Deportes, es la capacidad para integrar información proveniente de las ramas de la biomecánica y la fisiología. Esta integración es verdaderamente compleja, pues se deben comprender, analizar y sintetizar conceptos de biología, anatomía funcional, bioquímica y física aplicadas al movimiento humano. Por tal motivo, el propósito de este estudio de caso es el de mostrar una aproximación de cómo se abordaría el estudio de un implemento deportivo de venta en el mercado costarricense, de manera que el cuerpo estudiantil pueda observar la integración de conceptos biomecánicos y fisiológicos.

Se seleccionó el Skyrunner ${ }^{\circledR}$, un dispositivo compuesto por un resorte de fibra de vidrio y una estructura de aluminio que se coloca en las piernas y que permite movilizarse por medio de la caminata, 


\begin{abstract}
The intention of this case study was to analyze a sport artifact sold in the Costa Rican market, the Skyrunner ${ }^{\circledR}$, from the biomechanical and physiological point of view. For it, the biomechanics and physiological differences are studied when the Skyrunner ${ }^{\circledR}$ is and is not used. A man (20 years, 1.67 $m, 55 \mathrm{~kg}$ ) was the case of study. The analysis of the biomechanical variables was made by means of a force platform, with which the vertical force was obtained, the height, the jump flight time, vertical impulse and duration of the impulse phase. For the measurement of the physiological variables a metabolic car was used to evaluate the power consumption while the subject was exercised in an endless band during the heating, the exercise and the phase of recovery. In general, the use of the Skyrunner ${ }^{\circledR}$ caused a greater cost of energy that can be attributed to the requirement of mechanisms of balance and muscular tension that entail an important cost of energy. The height of the jumps with Skyrunner ${ }^{\circledR}$ was a greater by $50 \%$, although the vertical impulse was near a $15 \%$ less. The time of impulse was $27.5 \%$ the time of a normal jump and forces were generated almost three times greater. This denotes the great mechanical efficiency of the system that is able to keep and use greater amount of elastic energy accumulated in the structure, in comparison with that produced by the legs in an isolated form.
\end{abstract}

Key words: Methods, physiology, biomechanics, energy expenditure, jump. la carrera y saltos (fotografías 1 y 3 ). Tal y como cuando se adquiere una bicicleta o unos patines, la persona primero debe pasar por un periodo de aprendizaje motor para lograr el equilibrio y la tensión muscular necesarios para no caerse y así poder dominar los movimientos con el dispositivo. El aparato fue introducido en Costa Rica en el 2008 y se promociona como un implemento para la recreación, deporte aventura, el entretenimiento y el entrenamiento.

Uno de los atractivos del Skyrunner ${ }^{\circledR}$ (2008), tal y como lo promocionan en su sitio Web (www.skyrunnercostarica.com), es que se pueden saltar grandes distancias y alturas y, además, permite la posibilidad de "correr" a velocidades de hasta $40 \mathrm{~km} / \mathrm{h}$.

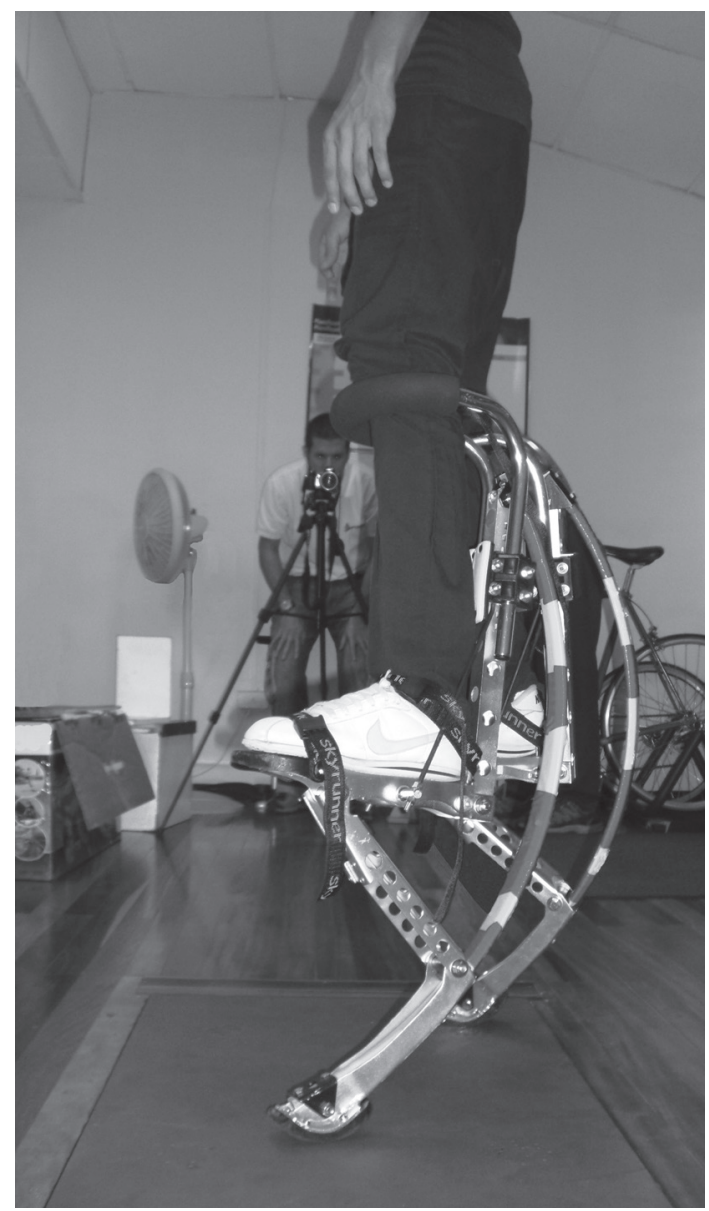

Fotografía 1. 


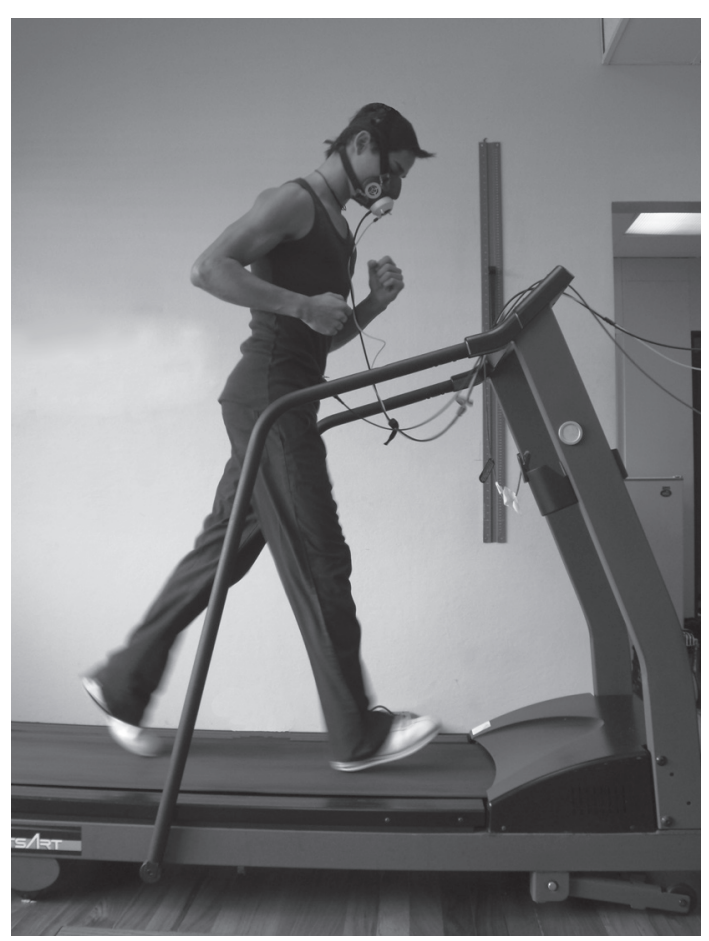

Fotografía 2.

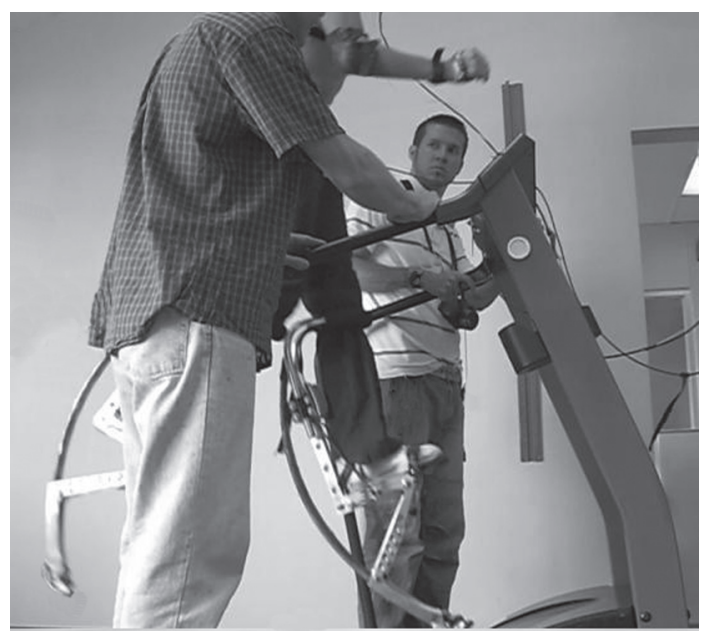

Fotografía 3.

Desde el punto de vista biomecánico, cuando una persona camina, corre o va a saltar, ejerce una fuerza contra la superficie, y ésta ejerce sobre la persona una fuerza igual y opuesta, llamada fuerza de reacción (Hamill y Knutzen, 2003). La magnitud de la componente vertical de la fuerza de reacción en conjunto con el impulso y la duración de la fase de impulso se relacionan directamente con la altura de un salto. Si la componente horizontal de la fuerza de reacción es la que se incrementa, entonces la tendencia va a ser producir un salto de longitud o distancia.

Entre mayor fuerza vertical se ejerza contra el piso, mayores posibilidades de impulsarse hacia arriba y obtener una altura mayor del salto. Además, si la duración de la fase de impulso es mayor, se produce un mayor impulso total. El impulso es una medida de lo que se requiere para cambiar el movimiento de un objeto. Así, durante un salto, se tiene que cambiar el estado de movimiento desde el reposo hasta una velocidad máxima del centro de masa en el momento del despegue. De esta manera, se desea obtener la mayor velocidad de despegue para que el salto sea de mayor altura (Adrian y Cooper, 1989).

Dadas las posibles ventajas biomecánicas que puede proporcionar este instrumento, se procedió a estudiar las diferencias biomecánicas y fisiológicas cuando no se usa y cuando sí se usa el Skyrunner ${ }^{\circledR}$. Por medio de un estudio biomecánico de las fuerzas de reacción sobre el piso y otras variables cinéticas se pretende explicar cómo es posible que se pueda lograr un mayor rendimiento en una persona que utiliza el dispositivo.

\section{Metodología}

\section{Participante}

Un hombre de 20 años de edad, 1,67 $\mathrm{m}$ de estatura y $55 \mathrm{~kg}$ de peso fue el caso de estudio. La superficie de área corporal fue de $1,6 \mathrm{~m}^{2}$, el índice de masa corporal fue de $19,7 \mathrm{~kg} / \mathrm{m}^{2}$, el porcentaje de grasa corporal por bioimpedancia eléctrica (BIA) fue de $7,3 \%$, y la frecuencia cardiaca máxima estimada fue de 200 latidos/minuto (220-edad). El participante lucía aparentemente sano y manifestó entrenar porrismo y gimnasia. Las pruebas se realizaron en el Laboratorio de Ciencias del Movimiento Humano -LACIMOV- de la Escuela de 
Educación Física de la Universidad de Costa Rica. La temperatura ambiental era de $25^{\circ} \mathrm{C}$ y la humedad relativa de $61 \%$.

\section{Materiales y procedimiento}

Variables biomecánicas. Para esta sección se utilizó una plataforma de fuerza (Bertec-Kistler ${ }^{\circledR}$, USA) que sirve para medir los tres componentes de la fuerza de reacción en el piso (vertical [Fz], mediolateral [Fy] y anteroposterior [Fx]) producidas durante actividades tales como la marcha (caminar, correr) o diversos tipos de saltos.

Los datos se recolectaron con una frecuencia de muestreo para las fuerzas de 1000 $\mathrm{Hz}$ y la señal se pasó por un filtro de baja frecuencia ("Double Butterworth Low Pass Filter") para eliminar ruido de la señal. El tiempo de recolección de datos se limitó a tres segundos para cada intento. En total se recolectaron tres repeticiones para cada condición (sin y con Skyrunner ${ }^{\circledR}$ ). En este caso, se utilizó para analizar las variables cinéticas del componente vertical de la fuerza de reacción, el tiempo de vuelo, el impulso vertical y la duración de la fase de impulso.

Para ilustrar el concepto de las variables medidas, se puede observar la curva representada en la Figura 1, la cual muestra la fuerza vertical aplicada a través del tiempo mientras el pie está en contacto con el suelo o la plataforma, generando una fuerza hacia abajo y recibiendo una fuerza de igual magnitud y opuesta en dirección, la cual produce el impulso hacia arriba. El impulso se puede expresar como el área bajo la curva de fuerza vs. tiempo (Hamill y Knutzen, 2003).

Así la altura de un salto depende del impulso, que a su vez depende tanto de la magnitud de la fuerza como el tiempo durante el cual se aplica la fuerza. Para un salto "contramovimiento", la fase de impulso se define desde el momento en que la fuerza llega a su valor mínimo por debajo del peso corporal antes de generar la fuerza máxima hasta el momento en que la fuerza se reduce hasta un valor mínimo
Figura 1

Componente vertical de la fuerza de reacción de un salto vertical con "contramovimiento"

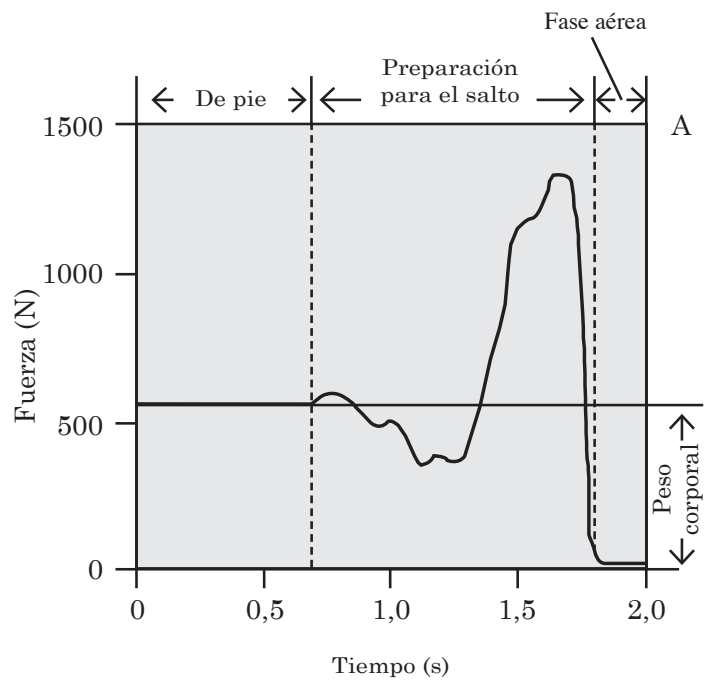

que representa el momento del despegue de la plataforma de fuerza, e iniciando la fase de vuelo. Luego de la fase de vuelo (donde desaparecen los valores de fuerza o cae a valores muy pequeños) viene la fase de aterrizaje, donde se amortigua el impacto de la caída (no mostrada en la Figura 1).

Por otro lado, desde el momento del despegue hasta el aterrizaje, el cuerpo se puede analizar de acuerdo a las ecuaciones de movimiento de un proyectil y está sujeto solamente a la fuerza y aceleración gravitacionales.

Variables fisiológicas. Para la medición del gasto energético se utilizó un carro metabólico marca COSMED ${ }^{\circledR}$, modelo Quark B ${ }^{2}$ (COSMED, Italia). Este analizador fue calibrado de acuerdo con los estándares de fábrica con gases de conocida concentración ( $5 \% \mathrm{CO}_{2}, 16 \% \mathrm{O}_{2}$ y balance de $\mathrm{N}_{2}$, Scott Medical, USA). Al participante se le colocó una mascarilla por donde los gases espirados eran recolectados por una línea de recolección que los llevaba hasta el analizador y así poder estimar la cantidad de oxígeno utilizado para oxidar diferentes sustratos energéticos (i.e., carbohidratos, proteínas) (Fotografía 4). 


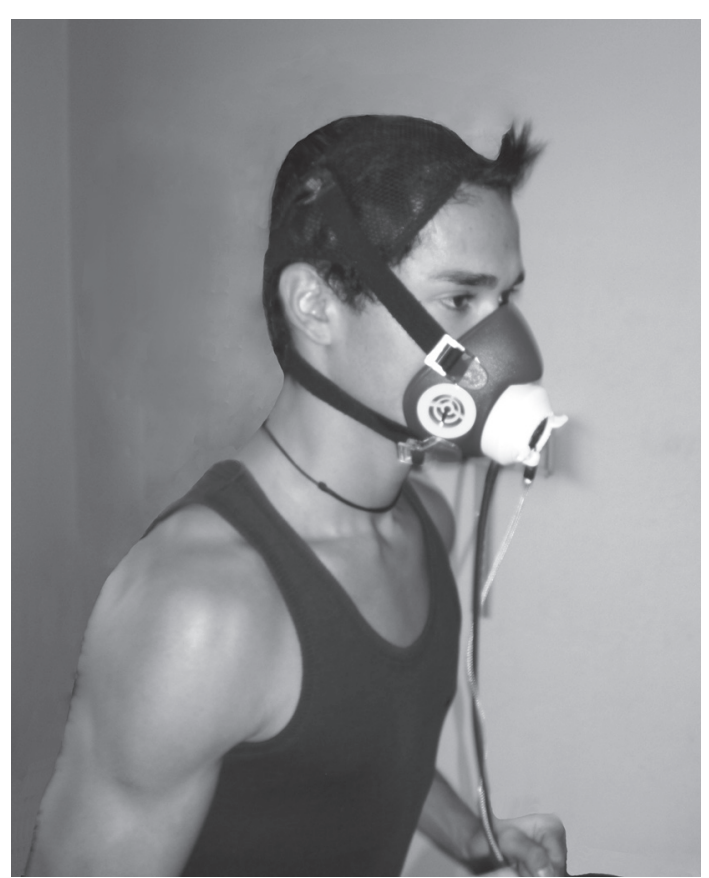

Fotografía 4.

Con esta información, se obtuvieron valores durante el calentamiento, durante el ejercicio y durante la fase de recuperación bajo dos condiciones distintas: a) sin el Skyrunner ${ }^{\circledR}$ (Fotografía 2) y b) utilizando el Skyrunner ${ }^{\circledR}$ (Fotografía 3). La fase de calentamiento consistió de caminar en una banda sin fin (SportsArt, USA), a $8 \mathrm{~km} / \mathrm{h}$ durante $3 \mathrm{~min}$. Posteriormente, se iniciaba la fase de ejercicio a $8 \mathrm{~km} / \mathrm{h}$ durante 5 min $y$, finalmente, se realizaba una fase de enfriamiento o vuelta a la normalidad a $4 \mathrm{~km} / \mathrm{h}$ durante $3 \mathrm{~min}$.

Durante todo momento, se midió la frecuencia cardiaca (latidos/minuto) de manera telemétrica (Polar ${ }^{\circledR}$, Finlandia), y se observaron los valores de frecuencia respiratoria (respiraciones/minuto), gasto energético (kcal), consumo de oxígeno $\left(\mathrm{VO}_{2}=\mathrm{mL} \mathrm{O}_{2} / \mathrm{kg}\right.$ peso corporal $/$ minuto), equivalentes metabólicos (METs) y porcentajes de carbohidratos y grasas utilizados.

\section{Análisis de datos}

Para las variables fisiológicas y metabólicas se obtuvieron los promedios para cada variable en la etapa de calentamiento, ejercicio y recuperación. Se calculó el porcentaje de la frecuencia cardiaca máxima obtenido en cada etapa de estudio y los porcentajes de sustratos utilizados durante el ejercicio. Por su parte, para las variables biomecánicas se obtuvieron los promedios para cada variable y se utilizaron fórmulas de proyectiles para calcular la altura del salto con base en la duración de la fase de vuelo.

\section{Resultados}

\section{Variables fisiológicas}

En la Tabla 1 se puede observar el comportamiento de las diferentes variables metabólicas cuando el participante no utilizó el Skyrunner ${ }^{\circledR}$ y cuando sí lo utilizó. Se puede observar que el gasto calórico expresado en Kcal fue similar en la etapa de calentamiento en ambas condiciones; sin embargo, durante los periodos de ejercicio y recuperación se obtuvieron mayores consumos energéticos al utilizar el Skyrunner ${ }^{\circledR}$.

Estos hallazgos se corroboran al observar el $\mathrm{VO}_{2}$ obtenido bajo ambas condiciones (Gráfico 1). En el panel de la izquierda se observa el $\mathrm{VO}_{2}$ en las tres etapas del estudio. En la primera parte, la línea ascendente indica el paso del reposo al calentamiento $(8 \mathrm{~km} / \mathrm{h})$. La línea irregular más horizontal representa el $\mathrm{VO}_{2}$ durante fase estable en ejercicio $(8 \mathrm{~km} / \mathrm{h})$. Finalmente, la línea descendente indica el $\mathrm{VO}_{2}$ en periodo de recuperación (a una velocidad de $4 \mathrm{~km} / \mathrm{h}$ ).

Al observar ambos paneles, se puede distinguir claramente que el $\mathrm{VO}_{2}$ fue distinto en ambas condiciones. Cuando el participante corrió sin el Skyrunner ${ }^{\circledR}$ el $\mathrm{VO}_{2}$ promedio en fase estable del ejercicio fue de $37,5 \mathrm{ml} / \mathrm{kg} /$ min. En cambio, en el panel de la derecha, se observa que la pendiente del $\mathrm{VO}_{2}$ es más inclinada (línea gruesa resaltada), lo que indica que se requiere de mayor cantidad de oxígeno $\left(\mathrm{VO}_{2}\right)$ para generar la energía necesaria para 
Tabla 1

Promedios de las variables fisiológicas y metabólicas medidas en cada etapa del estudio

\begin{tabular}{|l|c|c|c|c|c|c|}
\hline \multirow{2}{*}{ Variables } & \multicolumn{3}{|c|}{ Sin Skyrunner® } & \multicolumn{3}{c|}{ Con Skyrunner } \\
\cline { 2 - 7 } & $\begin{array}{c}\text { Calenta- } \\
\text { miento }\end{array}$ & Ejercicio & $\begin{array}{c}\text { Recupera- } \\
\text { ción }\end{array}$ & $\begin{array}{c}\text { Calenta- } \\
\text { miento }\end{array}$ & $\begin{array}{c}\text { Ejercicio } \\
\text { Recupe- } \\
\text { ración }\end{array}$ \\
\hline GCT (kcal) & 9,0 & 48,8 & 85,0 & 9,5 & 50,3 & 92,6 \\
\hline CHO (\%) & 81,4 & 92,4 & 98,5 & 94,4 & 99,5 & 96,2 \\
\hline Grasas (\%) & 18,6 & 7,6 & 1,5 & 5,6 & 0,5 & 3,8 \\
\hline VO ${ }_{2}(\mathrm{ml} / \mathrm{kg} / \mathrm{min})$ & 27,9 & 37,5 & 25,3 & 27,5 & 41,4 & 30,3 \\
\hline FC (lat/min) & 129 & 142 & 122 & 150 & 183 & 167 \\
\hline FC máx $(\%)$ & 64 & 71 & 61 & 75 & 92 & 84 \\
\hline FR (resp/min) & 25 & 28 & 32 & 35 & 53 & 48 \\
\hline
\end{tabular}

Abreviaturas:

GCT: Gasto calórico total; CHO: porcentaje de carbohidratos utilizados; $\mathrm{VO}_{2}$ : Consumo de oxígeno; FC: Frecuencia cardiaca; ${ }_{F C m a ́ x \%}$ : Porcentaje de la frecuencia cardiaca máxima; FR: Frecuencia respiratoria.

\section{Gráfico 1}

Consumo de oxígeno durante carrera en banda sin fin bajo dos condiciones de ejercicio, sin Skyrunner ${ }^{\circledR}$ (panel izquierdo) y con Skyrunner ${ }^{\circledR}$ (panel derecho)
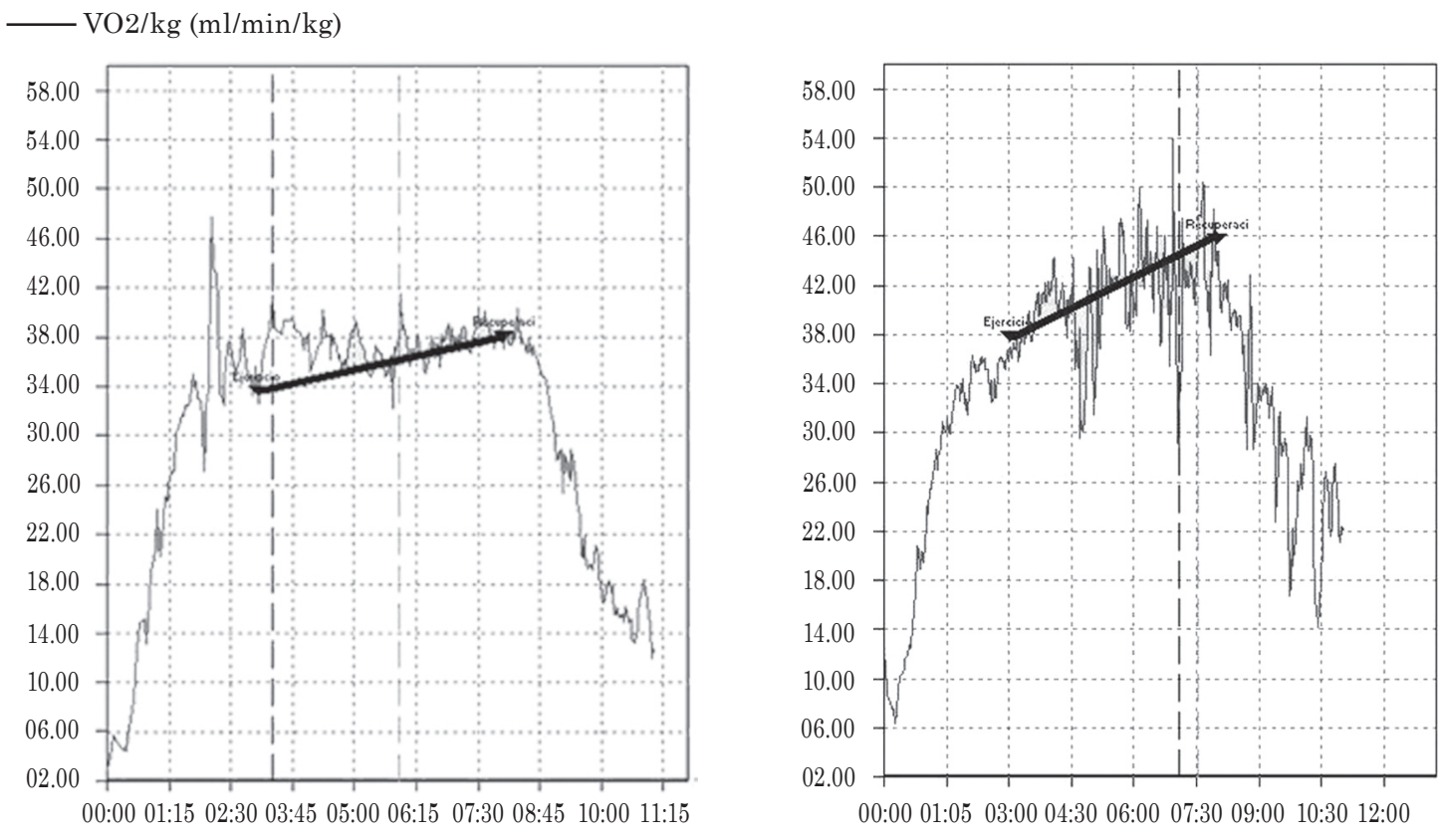
correr a $8 \mathrm{~km} / \mathrm{h}$ en la banda sin fin, que en términos relativos fue de $41,4 \mathrm{ml} / \mathrm{kg} / \mathrm{min}$. La pendiente de ambos gráficos es comparable pues los ejes $\mathrm{Y}(\mathrm{ml} / \mathrm{kg} / \mathrm{min})$ fueron construidos con la misma escala.

El aumento en el gasto energético y, principalmente, el consumo de carbohidratos y grasas utilizadas indican que, para esta persona en particular, el ejercicio realizado fue más de tipo anaeróbico (i.e., dependiente de la glucosa) que aeróbico (i.e., dependiente de las grasas). Esta afirmación se basa en la mayor cantidad de carbohidratos utilizados durante la prueba con el Skyrunner ${ }^{\circledR}$ en comparación a cuando realizó ejercicio sin el aparato. Sin embargo, en general, se puede afirmar que la utilización del Skyrunner ${ }^{\circledR}$ provocó un mayor gasto de energía, lo cual es obvio desde el punto de vista fisiológico ya que se requieren de mecanismos de equilibrio y tensión muscular que requieren de una importante cantidad de energía.

Variables biomecánicas. La Tabla 2 muestra los promedios para tres repeticiones de salto vertical sobre la plataforma de los valores de tiempo de vuelo $\left(\mathrm{t}_{\text {vuelo }}[\mathrm{s}]\right)$, altura del salto (h $[\mathrm{cm}]$ ), fuerza máxima desarrollada en la fase de impulso ( $\mathrm{F}_{\text {máx }}$ $[\mathrm{N}])$, impulso del salto (Imp $\left[\mathrm{N}^{*} \mathrm{~s}\right]$ ), y duración de la fase de impulso $\left(\mathrm{t}_{\mathrm{imp}}[\mathrm{s}]\right)$.

El Gráfico 2 muestra la curva para uno de los saltos del sujeto de este estudio, donde se pueden observar la técnica del salto "contramovimiento", presentando una disminución de la fuerza por debajo del peso del sujeto un instante antes de generar el impulso del salto y que representa el momento en que el sujeto se agacha para "coger impulso", como suele decirse comúnmente. Esta maniobra de agacharse resulta en lo que se llama el ciclo de estiramiento-acortamiento donde se acumula energía elástica principalmente en el conocido tendón de Aquiles, que luego se libera en el momento que las piernas se extienden.

El Gráfico 3 representa la curva de fuerza vs. tiempo para la condición "Con
Tabla 2

Promedios de variables biomecánicas

\begin{tabular}{|l|c|c|}
\hline \multicolumn{1}{|c|}{ Variables } & $\begin{array}{c}\text { Sin } \\
\text { Skyrunner }\end{array}$ & $\begin{array}{c}\text { Con } \\
\text { Skyrunner }\end{array}$ \\
\hline $\mathrm{t}_{\text {vuelo }}(\mathrm{s})$ & 0,55 & 0,69 \\
\hline $\mathrm{h}(\mathrm{cm})$ & 37,45 & 58,20 \\
\hline $\mathrm{F}_{\text {máx }}(\mathrm{N})$ & 1563,33 & 4117,00 \\
\hline $\operatorname{Imp}\left(\mathrm{N}^{*} \mathrm{~s}\right)$ & 550,40 & 476,14 \\
\hline $\mathrm{T}_{\text {imp }}(\mathrm{s})$ & 0,65 & 0,18 \\
\hline
\end{tabular}

Abreviaturas

$\mathrm{t}_{\text {vuelo }}$ : Tiempo de vuelo; h: Altura del salto; $\mathrm{F}_{\text {máx }}$ : Fuerza máxima desarrollada en la fase de impulso; Imp: Impulso del salto; $\mathrm{T}_{\mathrm{imp}}$ : duración de la fase de impulso.

Skyrunner ${ }^{\circledR}$, mostrando una forma bastante diferente, con una serie de pequeños saltos antes del salto mayor. El intervalo entre cada pequeño salto es el período de vuelo que sirve como fuente de energía potencial y cinética que se utiliza para "cargar" la hoja de fibra de vidrio con energía elástica que se acumula en la estructura cuando el sujeto "cae" y que luego es liberada al extenderse la pierna y la hoja de fibra de vidrio, impulsando de ese modo al sujeto hacia arriba.

Es además un gran reto para el sistema de control neuromuscular el poder mantenerse en una posición estática de pie debido a que el hecho de llevar puestos los "Skyrunners" resulta en una base de soporte de menor tamaño y una posición del centro de masa a mayor altura, produciendo una situación de menor estabilidad postural en comparación con la posición normal de pie o listo para ejecutar un salto.

\section{Discusión}

Se puede observar en la Tabla 2 que la altura del salto con Skyrunner ${ }^{\circledR}$ es aproximadamente un 50\% mayor que la altura sin Skyrunners ${ }^{\circledR}$, lo cual era de esperarse, aún en las condiciones en las cuales se realizaron las pruebas. Resulta 


\section{Gráfico 2}

Curva de fuerzas (F) vs. tiempo "sin Skyrunner ${ }^{\mathbb{B}}$ ", mostrando las fuerzas de reacción en "x", “y” y "z" durante un salto "contramovimiento" de una de las repeticiones

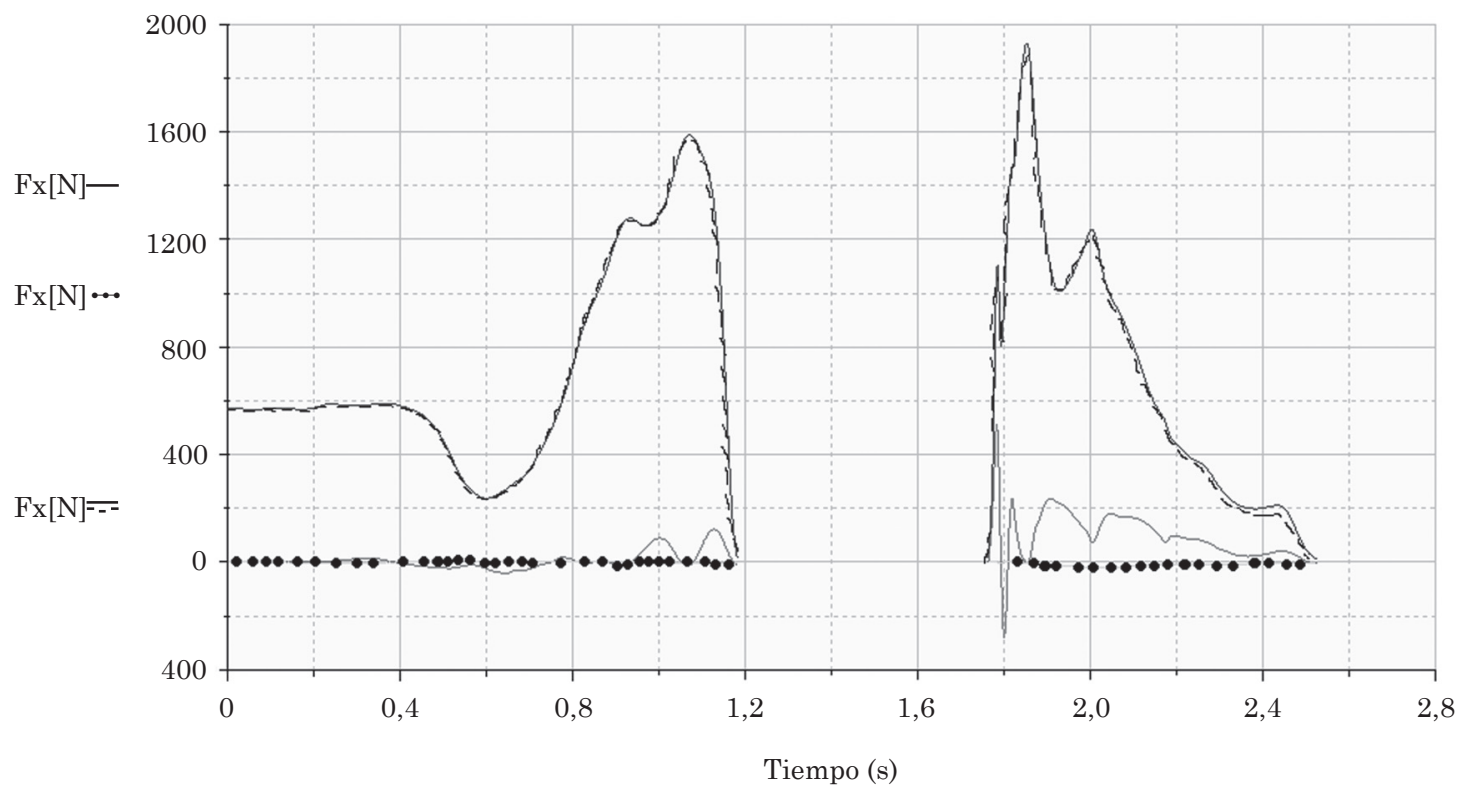

Gráfico 3

Curva de fuerza vs. tiempo “con Skyrunner" ${ }^{\circledR}$ de una de las repeticiones

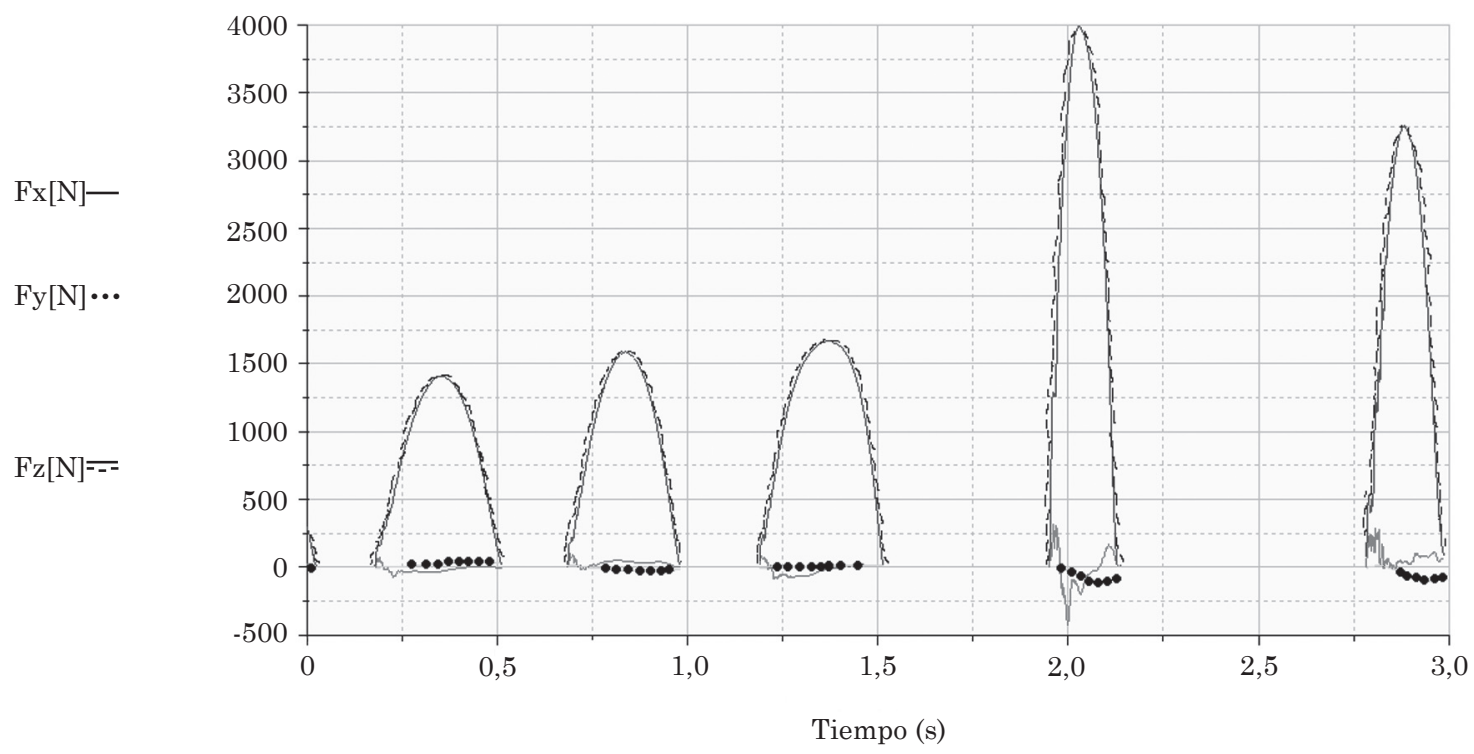

bastante complicado hacer saltos sobre el mismo lugar ya que los Skyrunners ${ }^{\circledR}$ no están diseñados para realizar estos saltos y son más bien eficientes para saltos de longitud y/o altura, que conllevan desplazamientos horizontales relativamente grandes (en comparación con saltos sin el equipo). 
Otra limitación importante fue la altura del cielo raso del laboratorio, que quedaba aproximadamente a un metro y medio sobre la cabeza del sujeto, limitando la posibilidad de realizar saltos para obtener máxima altura. Lo que resulta muy interesante es que a pesar de las limitaciones, se lograron saltos de mayor altura con los aparatos. Y aún más relevante es que los saltos fueron de mayor altura a pesar de que el impulso vertical fue cerca de un 15\% menor con los aparatos que sin ellos. Esto denota la gran eficiencia mecánica del sistema, el cual es capaz de guardar y utilizar mayor cantidad de energía elástica acumulada en la estructura en comparación con las piernas normales (Hamill y Knutzen, 2003). Es además notorio el hecho de que la fuerza de reacción vertical máxima contra el piso fue 2,63 veces mayor al utilizar los aparatos lo que también demuestra la posibilidad de acumular y utilizar energía elástica para incrementar la fuerza generada contra una superficie. Dicha energía elástica se genera por medio del mayor desplazamiento del centro de masa del sujeto cuando va cayendo o agachándose para tomar impulso, que deforma la estructura y "carga" de energía elástica dicha estructura que luego se libera al iniciar el movimiento hacia arriba.

El impulso depende tanto de la fuerza como del tiempo que se aplica dicha fuerza, así, se sabe que se genera mayor fuerza con los aparatos, por lo tanto para generar un impulso similar se requiere menor tiempo de aplicación de la fuerza (Adrian y Cooper, 1989). En este caso el tiempo de impulsión fue de un $27,5 \%$ del tiempo de un salto normal y se generaron fuerzas hasta casi tres veces mayores. Esto puede ser un factor importante para el entrenamiento neuromuscular donde la velocidad de estiramiento de las fibras musculares puede ser clave en adaptaciones para mejorar las cualidades reactivas del sistema musculoesquelético (Baechle y Earle, 2000).
Además, el hecho de que el tiempo de impulso y la posibilidad de generar aún mayores fuerzas pudo verse limitado en este caso por las circunstancias de que los saltos se realizaron sobre un espacio limitado y con altura limitada, y permite pensar en las posibilidades que pueden darse bajo otras circunstancias. Por ejemplo, si una persona se entrenara con los Skyrunners ${ }^{\circledR}$, no tuviera limitaciones de espacio o altura, y lograra un tiempo de impulso similar al de un salto normal sin aparatos, podría generar hasta cuatro veces el impulso que se obtuvo en las condiciones de la prueba, lo cual ayudaría a explicar cómo es que una persona puede saltar hasta 2 metros de altura o correr hasta una velocidad de $40 \mathrm{~km} / \mathrm{h}$.

Con base en los datos recolectados, se puede concluir que la utilización del Skyrunner ${ }^{\circledR}$ en el participante de este caso promovió un mayor gasto energético al compararlo con una carrera en banda $\sin$ fin sin los dispositivos. La mayoría de los sustratos para la producción de energía provinieron de carbohidratos, por lo que se puede concluir que el Skyrunner ${ }^{\circledR}$ promueve ejercicio predominantemente de tipo anaeróbico (Brooks, Fahey, White y Baldwin, 2000; McArdle, Katch y Katch, 2001).

El Skyrunner ${ }^{\circledR}$ resulta ser una máquina muy interesante desde el punto de vista biomecánico por sus enormes posibilidades de acumulación y utilización de energía elástica para producir grandes cantidades de trabajo. Además, resulta ventajoso a nivel del entrenamiento de las cualidades elásticas y de absorción de energía en las fibras musculares y los tejidos tendinosos por medio del principio de sobrecarga.

Por otro lado, es importante mencionar que el hecho de poder generar o absorber grandes cantidades de energía y fuerzas en las estructuras musculoesqueléticas por medio de uso de los Skyrunners ${ }^{\circledR}$ se convierte en un factor de riesgo para sufrir lesiones (en el sitio web del distribuidor se muestran consejos para reducir accidentes), 
especialmente durante los aterrizajes, por lo que se requiere que el usuario siga un proceso de aprendizaje motor (similar a cuando se "aprende" a utilizar patines o andar en bicicleta) y tenga ciertas cualidades mínimas de fuerza y equilibrio que le ayuden a prevenir posibles lesiones.

\section{Nota}

* Los profesores M.Sc. José Moncada Jiménez y M.Sc. Ing. Pietro Scaglioni Solano, no tienen vínculos ni intereses comerciales con la marca Skyrunner ${ }^{\circledR}$. Se agradece la colaboración de la Srta. Heidy Saavedra por el apoyo técnico durante la recolección de datos.

\section{Referencias bibliográficas}

Adrian, M. y Cooper, J. (1989). The biomechanics of human movement [La biomecánica del movimiento humano]. Indianapolis, IN: Benchmark Press, Inc.

Baechle, T. y Earle, R. (2000). Essentials of strength training and conditioning [Principios de acondicio- namiento y entrenamiento contra resistencia]. Champaign, IL: Human Kinetics.

Brooks, G. A., Fahey, T. D., White, T. P. y Baldwin, K. M. (2000). Exercise physiology, Human bioenergetics and its applications [Fisiología del ejercicio: Bioenergética humana y sus aplicaciones] ( $3^{\text {rd }}$ ed.). Mountain View, CA: Mayfield Publishing Co.

Hamill, J. y Knutzen, K. (2003). Biomechanical basis of human movement [Bases biomecánicas del movimiento humano]. Baltimore, MD: Williams \& Wilkins.

McArdle, W. D., Katch, F. I. y Katch, V. L. (2001). Exercise physiology: Energy, nutrition, and human performance [Fisiología del ejercicio: Energía, nutrición y rendimiento humano] ( $5^{\text {th }}$ ed.). Philadelphia, PA: Lippincott Williams \& Wilkins.

Skyrunner ${ }^{\circledR}$. (2008). Skyrunner ${ }^{\circledR}$ CostaRica. Consultado el 23 de julio del 2008 de http://www.skyrunnercostarica.com 